\title{
The Human Brain
}

\author{
Paul T E Cusack* \\ 23 Park Ave. Saint john, Canada \\ Corresponding author: Paul TE Cusack, 23 Park Ave, Saint john, NB, Canada
}

\begin{tabular}{|c|c|}
\hline ARTICLE INFO & ABSTRACT \\
\hline Received: 豐 October 13, 2020 & In this paper, we attempt to establish a mathematical foundation or set of equations \\
\hline Published: 㓞 October 29, 2020 & $\begin{array}{l}\text { for the human brain. We consider the brain as a black box, with internal energy and } \\
\text { an input and output. The input is the sensory information. The internal energy is the }\end{array}$ \\
\hline $\begin{array}{l}\text { Citation: Paul T E Cusack. The Human } \\
\text { Brain. Biomed J Sci \& Tech Res 31(3)-2020. } \\
\text { BJSTR. MS.ID.005108. }\end{array}$ & $\begin{array}{l}\text { We can see for a plot of most of the equations that there is a mathematical solution to the } \\
\text { human brain problem. Putting the brain and mind on a mathematical footing is beneficial } \\
\text { toward predicting its behaviour. }\end{array}$ \\
\hline
\end{tabular}

Keywords: Brain Formulas; Neurotransmitters; Hormones; Senses; Mind as Black Box

\section{Mini Review}

In this paper, we use the equations of the brain and mind to develop a mathematical model for the brain. Using energy methods, we consider the input from the senses, the internal energy from the neurotransmitters and the output from the motor nerves, and the hormones. We begin with the cache of the formulas that have already been developed for the brain in previous papers.

The formulas necessary for understanding the human brain include:
1) $t^{2}-t-1=E$
2) $\quad i=t^{2}$
3) $\quad V=i R$
4) $L=L n t+c^{2}$
5) $S E=E \cdot M$
6) $E=(1-\operatorname{Ln} t)^{\wedge} 7$
7) $M=\operatorname{Ln} t$
8) $E=1 / t$
9) $t=E^{2}+E-2$
10) $E=d H=S T d s+V d p+\sum \mu N$
11) $\sum$ Senses $=1 / \pi+\pi+4+\sqrt{G}+\sqrt{3}=1$

(Figure 1).

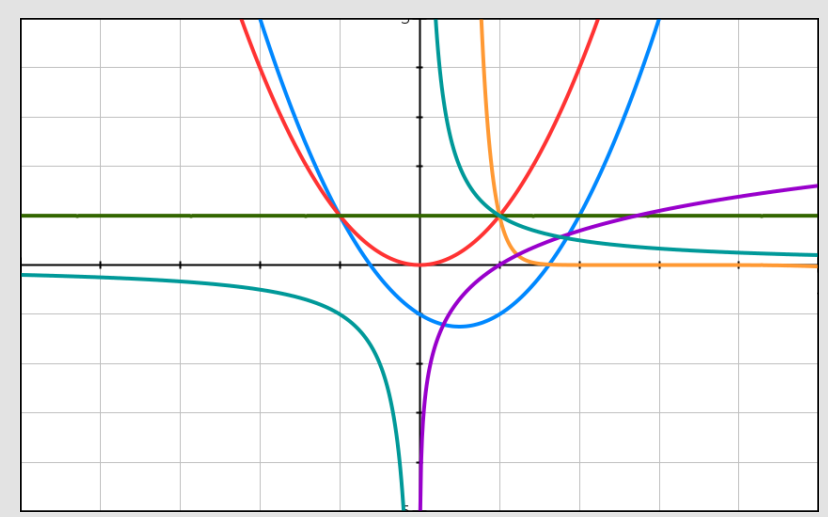

Figure 1: Plot of some of the Mind Equations. 
There are hundreds of neurotransmitters. The most important ones are:

- $\quad$ Acetylcholine (Ach)

- Norepinephrine

- $\quad$ Serotonin

- Dopamine

- $\quad$ Phenylethylamine (PEA)

- $\quad$ GABA

- Glutamate

- $\quad$ Substance P

- $\quad$ CGRP

- $\quad$ Neuropeptide Y

- Glycine

$t^{2}-t-1=E$

$(1-\operatorname{Ln} t)^{7}$

$t=1.3888$

$\mathrm{i}=\mathrm{t}^{2}$

$=1.3888^{2}$

$=1.929$

$(1-\ln 1.3888)^{7}$

$=0.0616$

$\mathrm{t}^{2}-\mathrm{t}-1=$

$(0.616)^{2}-(0.616)-1=-1.057=\mathrm{V}$

$\mathrm{V}=\mathrm{iR}$

$-105.7=1.929 R$

$\mathrm{R}=0.548$

$\mathrm{M}=\ln \mathrm{t}=\operatorname{Ln} 1.3888=328$

$\mathrm{E}=1 / \mathrm{t}=1 / 1.3888=1 / 7.2=\mathrm{SE}=(\mathrm{E} \cdot \mathrm{M})$

$\mathrm{SE}=(-1 / 8 \cdot 1 / 9)=1 / 72$

$t=E^{2}-E-2$

$=(1 / 7.2)^{2}+1 / 7.2-2$

$=654$

\section{Enthalpy}

$$
\begin{aligned}
& E=d H=S T d s+V d p+\sum \mu N \\
& d H=t(\Delta Q / T)+0+M c^{2}
\end{aligned}
$$

$98=\Delta Q+0+328 c^{2}$

$\Delta \mathrm{Q}=95.05=1 / 105.2 \sim \mathrm{V}$

$\mathrm{t}^{2}=\Delta \mathrm{Q}-\mathrm{dH}$

$\mathrm{t}^{2}=\mathrm{ST}-\mathrm{dH}$

$i=S T-d H$

$V=i R$

$=(\mathrm{ST}-\mathrm{dH}) \mathrm{R}$

$=(\mathrm{ST}-\mathrm{dH}) \mathrm{R}$

$1.052=(\Delta \mathrm{Q}-\mathrm{dH})(548)$

$1.052 / 0.548=\mathrm{i}=\mathrm{t}^{2}$

$=1.920$

t=1.3855 Cf. 1.388

$\mathrm{i}=1.920$

$=(\Delta \mathrm{Q}-\mathrm{dH})$

$192.0=95.05-\mathrm{dH}$

$\mathrm{dH}=96.85$

$t^{2}-t-1=E=E$

$(1)^{2}-1-1=-2$

$E=-2=-L$

$L=\operatorname{Ln} t+c^{3}$

$2=\operatorname{Ln} t+27$

$\operatorname{Ln} \mathrm{t}=-25$

$-\operatorname{Ln} \mathrm{t}=25$

$\mathrm{t}=-3.218$

$t=E^{2}+E-2$

$-3.218=E^{2}+E-2$

$E=-2.22222 ;-1.22222$

$\mathrm{t}=(1-\mathrm{SE} / \mathrm{E})^{7}$

$t=1-(-2 / 2.2222)=(1-1 /(1 / 9)=-8$

$\mathrm{E}=-1.25=$ Emin

$\mathrm{TE}=\mathrm{M}[0.15915]+$ Delta $Q$

$=1181 . .[.15915]+96.85$

$=115.6417=1 / \sin 60 \mathrm{deg}$.

We now consider the 5 most important neurotransmitters:

1) Dopamine $\mathrm{C} 8 \mathrm{H} 9 \mathrm{~N} 1 \mathrm{O} 2=138 \mathrm{amu}$ 
2) Serotonin $\mathrm{C} 10 \mathrm{H} 10 \mathrm{~N} 201=174 \mathrm{amu}$

3) GABA C4H6N102=100amu

4) Norepinephrine $\mathrm{C} 8 \mathrm{H} 10 \mathrm{~N} 103=168 \mathrm{amu}$

5) Glutamic Acid C5H7N104=145 amu

SUM=725 amu

$M=\operatorname{Ln} t$

$725=\operatorname{Ln} t$

$\mathrm{t}==2.0647$

$t^{\wedge} 2-t-1=1.198 \sim(1.2)$

$\mathrm{t}^{\wedge} 2-\mathrm{t}-1=\mathrm{E}$

$0.725^{\wedge} 2-0.725-1=-1.998 \sim(-1.2)$

$E=h \times$ freq

$=1 / \sin 60=6.626 \mathrm{t}$

$\mathrm{t}=5.738 \sim 1 \mathrm{rad}$.

12 pairs of cranial nerves and 31 pairs of Spinal Nerves.12/ $\{31 / \operatorname{Ln} 31]=1.329$

$1.329 \times 2=2.658 \sim 2.66=\mathrm{SF}$

\section{Planck's Equation}

$E=h v$

$\mathrm{dH}=\mathrm{E}=1 / \sin 60^{\circ}$

$2 \times 1 / \sin 60^{\circ}=2(115.47)=2.3094 \sim 231$

$\operatorname{Ln}(23.094)=8369=1 / 1.1947 \sim 1 / 12$

\section{Sight and Sound}

\section{Sight}

$E=h v$

$=(6.626)(380 \mathrm{~nm}-720 \mathrm{~nm})=2.517-4.770=2.25$

\section{Sound}

$E=h v=(6.626)(20-20,000)=132.5-132,500=132.385$

$2.25-132.385=134.75$

$134.75 \times 2=26.95 \sim 27=c^{3}$

$\mathrm{L}=2=(134.75)(2)=27+\mathrm{Ln} \mathrm{t}$

$0=\operatorname{Ln} t$

$\mathrm{t}=1$

$0=\operatorname{Ln} \mathrm{t}$ $t=1$

(Table 1).

Table 1.

\begin{tabular}{|c|c|c|}
\hline Hormone & Formula & amu \\
\hline Adrenalin & C9H13NO3 & 183.2 \\
\hline Thyroid & C15H1144NO4 & 1427.8 \\
\hline Luteinizing & C59H83N17017 & 1302.4 \\
\hline FSH & C79H125N11023S & 1741 \\
\hline Growth Hormone & C77H109N21019S & 1664.9 \\
\hline Melanocyte & C18H10N204 & 3183 \\
\hline Sum & & 9502 \\
\hline & X 6.026 atm /mole & $57.23=1 \mathrm{rad}$ \\
\hline
\end{tabular}

$M=L n t$

$1=\operatorname{Ln} t$

$\mathrm{t}=\mathrm{e}^{\wedge} 1$

$\mathrm{E}=1 / \mathrm{e}^{\wedge} 1=\mathrm{e}^{\wedge}-1$

\section{Input=Senses}

$\mathrm{V}=\mathrm{iR}=\left(1 / \mathrm{c}^{\wedge} 2\right)(1)$

$105.7\left(c^{\wedge} 2\right)=R$

$\mathrm{R}=950$

\section{Internal}

$\mathrm{t}^{\wedge} 2-\mathrm{t}-1=\mathrm{E}=1.500$

$\mathrm{t}^{\wedge} 2-\mathrm{t}-2.5=0$

$\mathrm{t}=21586 ; 115.83$

\section{Output}

$E=d H=1 / \sin 60=0.866$

Input-Output $=1 / \mathrm{c}^{\wedge} 2-0.866=-0.7547$

Input -Output + Internal $=-0.755+1.5=745$

$T E=M[0.15915]+$ deltas $Q$

$0.745=\mathrm{M}[0.15915]+96.05$

$M=1354 \sim 1350=$ Mass of Human Brain

$\mathrm{M}=\mathrm{Ln} \mathrm{t}$

$1.354=\operatorname{Ln} t$

$\mathrm{t}=26=$ Poisson's Ratio

(Figure 2). 


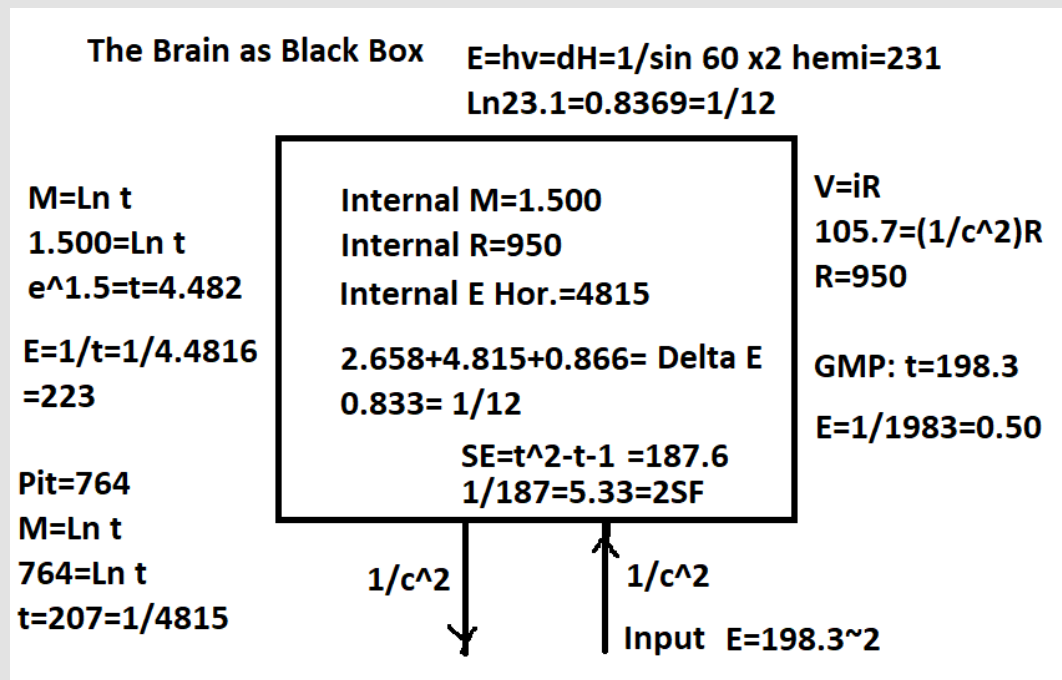

Figure 2.

The sense of smell is used by animals to communicate, for mating, and for food detection and predator detection. Smell is tied in with the orbitofrontal cortex to be used in value judgement. Smell is the only sense that does not pass through consciousness but is directly hardwired to the limbic system. We can therefore smell before we are consciousness of it likely due to the flight or fight response. Its parameters are equal to the universal parameters including: Energy (Emin=-1.247); time (eigenvector $=\sqrt{3}$ ); Mass ( $=\operatorname{Ln} \mathrm{t}$ ); the determinant $|\mathrm{D}|=4$ ) and the gravitational constant $(G=2 / 3=0.666)[1]$.

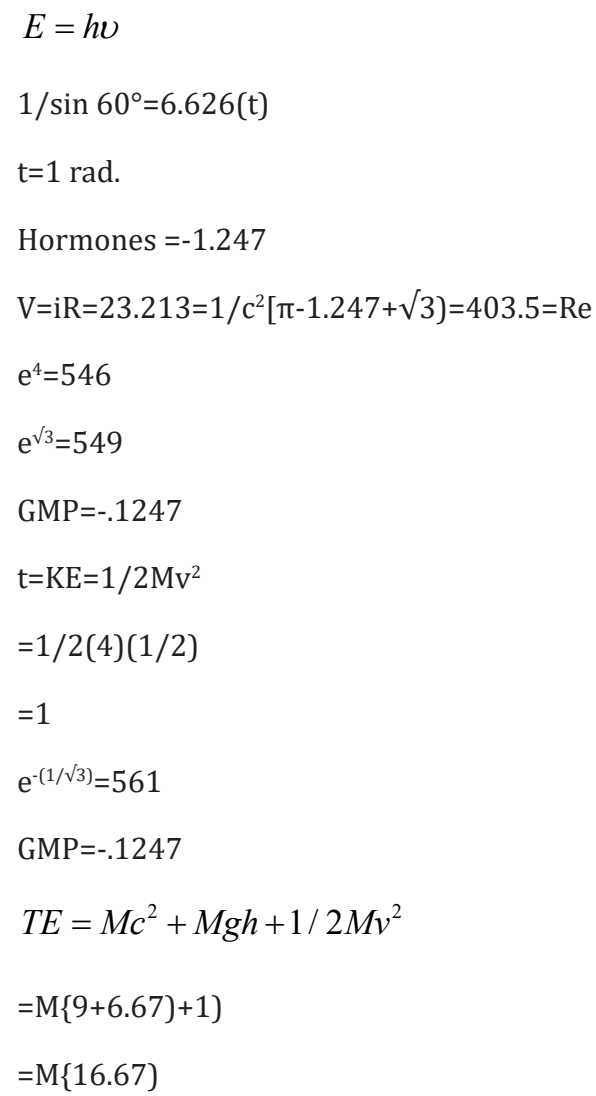

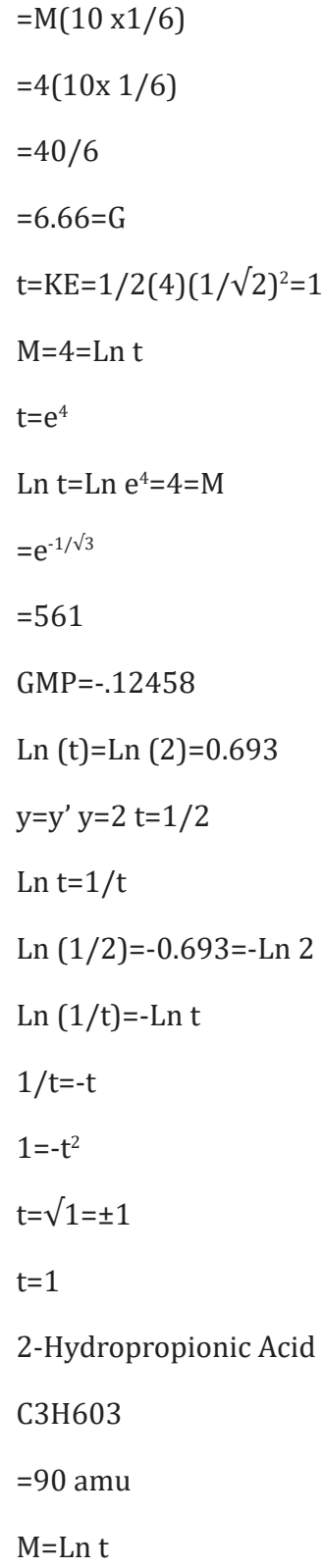


$90=\mathrm{M}=\mathrm{Ln} \mathrm{t}$

$\mathrm{t}=1.2204$

$E=819.4$

\section{Neurotransmitters}

GABA 100amu

Glutamic Acid 145 amu

Aspartic Acid 133.103 amu

Glycine $75.067 \mathrm{amu}$

$\Sigma 453.17 \times 6.023=2729 \mathrm{gm}$

$M=\operatorname{Ln} t=2729=\operatorname{Ln} t$

$\mathrm{t}=3.303$

$E=3.03$

$\mathrm{E}_{\mathrm{L} . \mathrm{A} .}-\mathrm{E}_{\mathrm{N} . \mathrm{T} .}=8194-3.03=0.816 .4=\sqrt{ }(2 / 3)=$ taste

$\mathrm{Na}^{+}$

$\mathrm{K}^{+}$

$\mathrm{Cl}^{-}$

Pr- 359

$\left(\mathrm{NO}_{3}^{-}\right.$;

$\mathrm{H}_{2} \mathrm{~S}$;

$\mathrm{CO})$

$\mathrm{Ca}^{+2}$

Vit. D 416

$\Sigma=775.1$

$+\mathrm{NT}=725=1500.1$

$\mathrm{V}=\mathrm{d} / \mathrm{t}$

$11.027=10 \mathrm{~m} / \mathrm{t}$

$\mathrm{t}=10 / 11.027=906.8=$ Minimum Time to recahrge $\sim 1 \mathrm{msec}$

$\mathrm{P}=\mathrm{i}^{2} \mathrm{R}$

$\mathrm{i}=\mathrm{t}^{2}=0.9068^{2}$

$\mathrm{P}=0.906 .84(950)$

$=676(950)$

$=6.422$

$\mathrm{P}=\mathrm{Et}$

$6.422=\mathrm{E}(906.8)$
$\mathrm{E}=708 \sim 1 / \sqrt{2}$

$\mathrm{P}=1 / 0.1557$

$1 / \sqrt{\mathrm{P}}=\sqrt{(1 / 0.1557)=1.247=\mathrm{E}}$

$\sqrt{\mathrm{P}}=1 / 1247=8.02=\mathrm{t}$

Universal Parametric Equation:

$\left[\csc 60^{\circ} ; 3\right]=[E ; t]$

Brain:

$[1 ; 1]=[\mathrm{E} ; \mathrm{t}]$

$b r_{\text {ain }}=U_{\text {niverse }}$

$\chi(1 / \sqrt{3})=1 / \sin 60^{\circ}$

$\chi=2.0000$

$\psi t_{\text {brain }}=t_{\text {universe }}$

$\psi(1)=$

$\psi=3$

$\chi / \psi=0.666=\mathrm{G}$

A plot of these two date points yields a linear equation:

$m=$ rise $/$ run $=\left[\left(1 / \sin 60^{\circ}\right)-1\right] / 3$

$=0.051578$

$\sim 0.052$

$b=1-0.052=947$

$y=0.052 x+948$

$E=0.052 t+948$

$\mathrm{E}=\mathrm{t}=1$

$\mathrm{dE} / \mathrm{dt}=0.052$

$=\left(\csc 60^{\circ}-1\right)$

$t=[E-948] / 0.052$

$\mathrm{dt} / \mathrm{dt}=1=[\mathrm{E}-948] / 0.052$

$\mathrm{E}=899=\mathrm{c}^{2}$

$t^{2}-t-1=-.052$

$\mathrm{t}^{2}-\mathrm{t}-1.052 \mathrm{mV}=0$

$t=1641 ; 641$

$E=609 ; 1.5$

(Figure 3). 


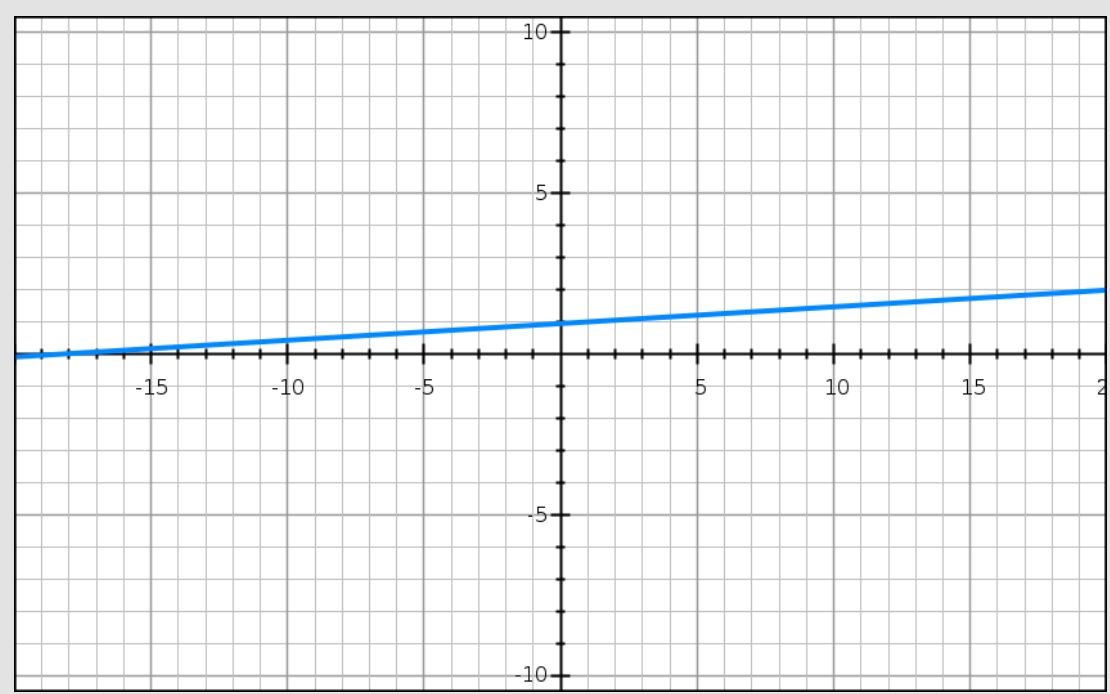

Figure 3.

\section{Conclusion}

We see that the brain can be modelled by 11 formulas. They converse on: $\mathrm{E}=1 ; \mathrm{t}-=1$ and $\mathrm{E}=0$; $\mathrm{t}=1$; and $\mathrm{E}=-1, \mathrm{t}=1$.

\section{ISSN: 2574-1241}

DOI: $10.26717 / B J S T R .2020 .31 .005108$

Paul T E Cusack. Biomed J Sci \& Tech Res

(C) (i) This work is licensed under Creative

Submission Link: https://biomedres.us/submit-manuscript.php

\section{References}

1. Carter Rita (2019) The Human Brain Book. Penguin Random House, USA.

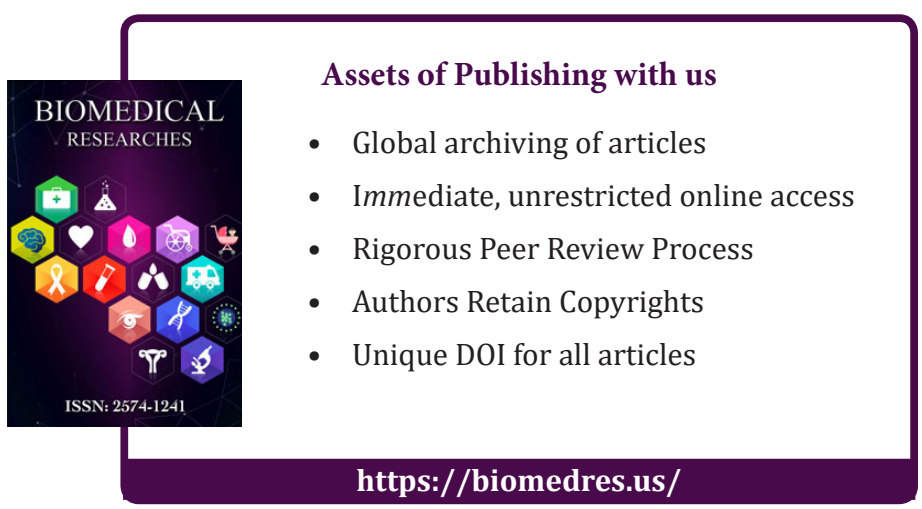

